\title{
The Consistent Estimators for Stationary Gaussian Statistical Structures in Hilbert Space of Measures
}

\author{
Z. Zerekidze, L. Aleksidze, L. Eliauri \\ Gori University, Gori, Georgia.
}

\begin{abstract}
The present theory a consistent estimators of the parameters of stationary Gaussian statistical structures in Hilbert space of measures can be used, for example, in the reliability predication of different engineering designs. In the paper there are discussed Gaussian stationary statistical structures $\left\{E, S, \mu_{i}, i \in I\right\}$ in Hilbert space of measures. We prove necessary and sufficient conditions for existence of such estimators in Hilbert space of measures.
\end{abstract}

Keywords: consistent estimators, orthogonal, weakly separable, strongly separable statistical structures. Ceassificationcocles 62HO5, 62H12

\section{Introduction}

Let there is given $(E, S)$ measurable space and on this space there given $\left\{\mu_{i}, i \in I\right\}$ family of probability measures definided on $\mathrm{S}$, the I set of parameters.

Let bring some definition (see [1]-[11]).

Definition 1. A statistical structure is called object $\left\{E, S, \mu_{i}, i \in I\right\}$ where $i \in I$ some parameter associated with probability measure $\left\{\mu_{i}, i \in I\right\}$, I set of parameters.

Definition 2. A statistical structure $\left\{E, S, \mu_{i}, i \in I\right\}$ is called orthogonal (singular) if $\mu_{i}$ and $\mu_{j}$ are orthogonal for each $i \neq j, \quad i \in I, \quad j \in I$.

Definition 3. A statistical structure $\left\{E, S, \mu_{i}, i \in I\right\}$ is called weakly separable if there exists family Smeasurable sets $\left\{X_{i}, i \in I\right\}$ such that relations are fulfilled: $(\forall i)(\forall j)(i \in I \& j \in I) \Rightarrow \mu_{i}\left(X_{j}\right)=\left\{\begin{array}{l}1, \text { if } i=j \\ 0, \text { if } i \neq j\end{array}\right.$.

Definition 4. A statistical structure $\left\{E, S, \mu_{i}, i \in I\right\}$ is called separable if there exist family S-measurable sets $\left\{X_{i}, i \in I\right\}$ such that relation is fulfilled:

1. $(\forall i)(\forall j)(i \in I \& j \in I) \Rightarrow \mu_{i}\left(X_{j}\right)= \begin{cases}1, & \text { if } i=j \\ 0, & \text { if } i \neq j\end{cases}$

2. $(\forall i)(\forall j)(i \in I \& j \in I) \Rightarrow \operatorname{card}\left(X_{i} \cap X_{j}\right)<c$,

where $\mathrm{c}$ denotes power continuum.

Definition 5. A statistical structure $\left\{E, S, \mu_{i}, i \in I\right\}$ is called strongly separable if there exists disjoint family S-measurable sets $\left\{X_{i}, i \in I\right\}$ such that the relation are fulfilled: $(\forall i)(\forall j) \rightarrow \mu_{i}\left(X_{i}\right)=1$.

Remark 1. A strong separable there follows weakly separable. From separable follows weakly separable. From weakly separable there follows orthogonal but not vice versa. (see [1]-[4])

Example 1. Let $E=[0,1] \times[0,1]$ and $\mathrm{S}$ be Borel $\sigma$-algebra of parts of E. Take the S-measurable sets $X_{i}=\{(x ; y) 0 \leq x \leq 1, \quad y=i, \quad i \in(0,1]\}$.

Let $l_{i}, i \in(0,1]$ be are linear Lebesgue probability measures on $X_{i}, i \in(0,1]$ and $l_{0}$ Lebesgue plane probability measures on $E=[0,1] \times[0,1]$. Then the statistical structure $\left\{E, S, l_{i}, i \in[0,1]\right\}$ is orthogonal, but not weakly separable. 
Remark 2. Z. Zerekidze proved in ZF theory (see [8]) for the statistical structure $M=\left\{E, S, \mu_{i}, i \in N\right\}$ where $\mathrm{N}$ set at natural numbers, orthogonality, weak separability, separability and strong separability equivalent concepts.

Let be I set of parameters and B(I) $\sigma$-algebra of subsets of I which contains all finite subsets I.

Definition 6. A statistical structure $\left\{E, S, \mu_{i}, i \in I\right\}$ will be said to admit a consistent estimators of parameter $i \in I$ if there exists at least one measurable map $\delta:(E, S) \rightarrow(I, B(I))$ such that $\bar{\mu}_{i}\{(x: \delta(x))=i\}=1, \quad \forall i \in I$.

We denote by $\bar{\mu}_{i}$ be completion of measures $\mu_{i}$ and $\operatorname{dom} \bar{\mu}_{i}$ the $\sigma$-algebra of all measurable subsets of $\mathrm{E}$. The Definite 6 is conducted Z. Zerekidze.

Let $M^{\sigma}$ be a real linear space of all alternating finite measures on $\mathrm{S}$.

Definite 7.A lineal subset $M_{H} \subset M^{\sigma}$ is called a Hilbert space of measures if:

1) One can introduce on $M_{H}$ a scale product $(\mu, v), \mu, v \in M_{H}$ is the Hilbert space and every mutually singular measures $\mu$ and $v, \mu, v \in M_{H}$, the scale product $(\mu, v)=0$;

2) If $v \in M_{H}$, and $|f(x)| \leq 1$ then $v_{f}(A)=\int_{A} f(x) v(d x) \in M_{H}$, where $f(x)$ is S-measurable real function and $\left(v_{f}, v_{f}\right) \leq(v, v)$;

3) If $v_{n} \in M_{H}, v_{n}>0, v_{n}(E)<+\infty, n=1,2, \ldots \quad$ and $\quad v_{n} \downarrow 0 \quad$ then for any $\mu \in M_{H} \lim _{n \rightarrow \infty}\left(v_{n}, \mu\right)=0$.

Remark 3.The definition ans construction of the Hilbert space of measures is studied Z. Zerakidze (see[7]).

Remark 4. A. Skorokhod was introduced definition a consistent estimators of parameters (see [1]).

Definition 8. A statistical structure $\left\{E, S, \mu_{i}, i \in I\right\}$ will be said to admit a consistent estimators of parameters $i \in I$ if there exists measurable map $\delta:(E, S) \rightarrow(I, B(I))$ such

that $\mu_{i}\{x: \delta(x)=i\}=1, \forall i \in I$. From this definition 8 follows that if statistical structure $\left\{E, S, \mu_{i}, i \in I\right\}$ admitting a consistent estimators of parameter $i \in I$, the it is clear that this statistical structure is strongly separable, but not vice versa (see [6]).

Z. Zerakidze constructed example (see [6]) that there is a strongly separable statistical structure which have no consistent estimators. From definition 8 it can be illustrated by the following example.

Example 2. Let $\mathrm{R}$ denote a numerical axis. Let $\mathrm{B}(\mathrm{R})$ be an usual Borel $\sigma$-algebra at this axis. Hence we shall consider a measurable space $(\mathrm{R}, \mathrm{B}(\mathrm{R}))$. Define a famile of Borel probability measures $\left(\mu_{x}\right)_{x \in R}$ on $\mathrm{B}(\mathrm{R})$, Let $f: R \rightarrow R$ denote some bijective into mapping at the axis $\mathrm{R}$ which is Borel non-measurable. Then, as we know, the inverse mapping $f^{-1}$ will also be Borel non-measurable. For every point $x \in R$ put $\mu_{x}(X)=\left\{\begin{array}{l}1, \text { if } f(x) \in X \\ 0, \text { if } f(x) \notin X\end{array}\right.$ where $\mathrm{X}$ passes through the $\sigma$-algebra $\mathrm{B}(\mathrm{R})$. In other words, the measure $\mu_{x}$ coincides with the Borel measure of Dirac, which is concentrated at the point $f(x)$. It can be easily seen that the statistical structure $\left\{R, B(R), \mu_{x}, x \in R\right\}$ is strongly separable, we can show that this statistical structure has no consistent estimator. From definition 8 assume the opposite: let $\mathrm{g}$ be a consistent estimator for the statistical structure. Then $\mu_{x}(\{y: g(y)=x\})=1, \quad \forall x \in R$.

The last equality means that $f(x) \in\{y: g(y)=x\}$ Hence, we have $g(f(x))=x, \forall x \in R$. This immediately implies that $g=f^{-1}$ and thus, the mapping is Borel non-measurable. This contradiction shows that no consistent estimator exists for the statistical structure $\left\{R, B(R), \mu_{x}, x \in R\right\}$ from definition 8 . 


\section{The Consistent Estimators}

In our work we will use definition 6 .

Let $\xi_{i}(t, w)=Q_{i}(t)+\Delta(t, \omega), t \in T \subset R, \forall i \in I$ Gaussian real stationary processes, where $\mathrm{T}$ be closed bounded subset of $\mathrm{R}$. with zero means $E(\Delta(t, \omega))=0, E\left(\xi_{i}(t, \omega)\right)=Q_{i}(t), t \in T, \forall i \in I$ and correlation function $E(\Delta(t, \omega) \Delta(k, \omega))=R(t-k$. $)$ Let $\left\{\mu_{Q_{i}}, i \in I\right\}, \quad \operatorname{card} I=C$ be the corresponding probability measures given on $\mathrm{S}$ and $f_{i}(\lambda), \lambda \in R, \forall i \in I$ be spectral densities such that relations are fulfilled: $\left(1+\lambda^{2}\right)^{-N} . k_{i} \leq f_{i}(\lambda) \leq C_{i}\left(1-\lambda^{2}\right)^{-N}, i \in I$ where $k_{i}$ and $C_{i}, i \in I$ are positive constants.

We shall assume that the functions itself or its derivatives satisfies conditions: $\int_{-\infty}^{+\infty}\left[Q_{i}^{(m)}(t)\right]^{2} d t=\infty \quad \forall i \in I \quad m=0,1,2, \ldots, n$.

Then the corresponding probability measures $\mu_{Q_{i}}(t)$ and $\mu_{Q_{j}}(t)$ are pairwise orthogonal $\forall i, j \in I$ (see [5], [11]) and $\left\{E, S, \mu_{Q_{i}}, i \in I\right\}, \operatorname{cardI}=C$ are Gaussian orthogonal stationary statistical structures.

Next, we consider S-measurable $g_{i}(x), \forall i \in I$ functions, such that $\sum_{I_{1}} \int_{E}\left|g_{i}(x)\right|^{2} \mu_{Q_{i}}(d x)<\infty$, where $I_{1} \subset I$ a countable subsets in I. Let $M_{H}$ the set measures defined by formula $v(B)=\sum_{i \in I_{1}} \int_{B} g_{i}(x) \mu_{Q_{i}}(d x), \quad$ define a scalar product by formula $\left(v_{1}, v_{2}\right)=\sum_{i \in I_{1} \cap I_{2}} \int g_{i}^{1}(x) g_{i}^{2}(x) \mu_{Q_{i}}(d x)$ where $I_{1} \subset I, I_{2} \subset I$ a countable subsets in I.

1. We will show $M_{H}$ is Hilbert space Let $\psi_{n}(B)=\sum_{i \in I_{n}} \int_{B} g_{i}^{m}(x) \mu_{Q_{i}}(d x)$,

Where $I_{n} \subset I, n=1,2, \ldots$ a countable subsets in $\mathrm{I}$ and $\psi_{n}$ is fundament sequence in $M_{H}$. Let $I^{\prime}=\bigcup_{n=1}^{\infty} I_{n}, \operatorname{card}^{\prime}<c$. So the Gaussian orthogonal statistical structure $\left\{E, S, \mu_{Q_{i}}, i \in I\right\}$, is strongly separable statistical structure (see remark 2), then instead of this functions $g_{i}^{n}(x)$ we take functions $g_{i}^{n}(x) I_{C_{i}}(x)\left(C_{i} \cap C_{j}=\varnothing\right)$, then $\psi_{n}(B)=\sum_{i \in I^{\prime}} \int_{B \cap C_{i}} g_{i}^{n}(x) \mu_{Q_{i}}(d x), \forall n \in N$.

Let $g_{I^{\prime}}^{n}(x)=\sum_{i \in I^{\prime}} g_{i}^{n}(x), \quad \mu_{I^{\prime}}(d x)=\sum_{i \in I^{\prime}} \mu_{Q_{i}}(d x)$,

It is clear, that

$\left\|\psi_{n}-\psi_{m}\right\|^{2} \leq \int\left|g_{I^{\prime}}^{n}(x)-g_{I^{\prime}}^{m}(x)\right|^{2} \mu_{I^{\prime}}(d x)$.

As well as $L^{2}\left(\mu_{I^{\prime}}\right)$ space is complete space, then exists such function $g_{I^{\prime}}(x)$, that $\int g_{I^{\prime}}^{2}(x) \mu_{I^{\prime}}(d x)<\infty, \int\left|g_{I^{\prime}}^{n}(x)-g_{I^{\prime}}(x)\right|^{2} \mu_{I}(d x) \rightarrow \infty, n \rightarrow \infty$.

Let $\psi_{n}(B)=\sum_{i \in I^{\prime}} \int_{B} g_{I^{\prime}}(x) I_{C_{i}}(x) \mu_{Q_{i}}(d x), \quad\left\|\psi_{n}-\psi\right\| \rightarrow 0, n \rightarrow \infty$.

2. If $v(B)=\sum_{i \in I_{0}} \int_{B} g_{i}(x) \mu_{Q_{i}}(d x)$, then $v_{f}(B)=\int_{B} f(x) v(d x)=\sum_{i \in I_{0}} \int_{B} f(x) g_{i}(x) \mu_{Q_{i}}(d x), \quad$ and so $|f(x)| \leq 1$, then $\left(v_{f}, v_{f}\right)=\sum_{i \in I_{0}} \int\left|f(x) g_{i}(x)\right|^{2} \mu_{Q_{i}}(d x) \leq \sum_{i \in I_{0}} \int\left|g_{i}(x)\right|^{2} \mu_{Q_{i}}(d x)=(v, v)$

3. Let $v=\sum_{i \in I_{1}} \int g_{i}(x) \mu_{Q_{i}}(d x), \quad \mu=\sum_{i \in I_{2}} \int f_{i}(x) \mu_{Q_{i}}(d x), \quad$ and $\mu \perp v$. 
Let $I_{3}=I_{1} \cup I_{2}$ and $\mu_{Q_{i}}\left(C_{j}\right)=\left\{\begin{array}{l}1, \text { if } i=j \\ 0, \quad \text { if } i \neq j\end{array} \quad i, j \in I_{3} C_{i} \cap C_{j}=\varnothing i \neq j . \quad\right.$ As $v \perp \mu \Rightarrow \sum_{i \in I_{3}} g_{i}(x) f_{i}(x)=0$ almost everywhere with respect $\mu_{I_{3}}$ and $(v, \mu)=\int \sum_{i \in I_{3}} g_{i}(x) f_{i}(x) \mu_{Q_{i}}(d x)=0$.

4. Let $v_{n} \in M_{H}, v_{n} \geq 0, v_{n} \downarrow 0, v_{n}(E)<\infty$, then if $v_{n}(B)=\sum_{i \in I_{n}} \int g_{i}^{n}(x) \mu_{Q_{i}}(d x) \in M_{H}, \forall n \in N$ can be considered $g_{i}^{n} \downarrow 0$ and $v_{n}(B)=\sum_{i \in I^{\prime}} \int_{B} g_{i}^{n}(x) \mu_{Q_{i}}(d x),\left(v_{n}, v_{n}\right)=\int \sum_{i \in I^{\prime}}\left|g_{i}^{n}(x)\right|^{2} I_{C_{i}}(x) \mu_{Q_{i}}(d x)$ and $\left(v_{n}, \psi\right) \rightarrow 0$.

We will show that $M_{H}$ is Hilbert space of measures. The following theorem has also been proved in this paper (see [7]).

Theorem 1. Let $M_{H}$ is Hilbert space of measures and $M_{H}$ is the straight sum of Hilbert spaces $H_{2}\left(\mu_{Q_{i}}\right)$, so $M_{H}=\underset{i \in I}{\oplus} H_{2}\left(\mu_{Q_{i}}\right)$,

where $H_{2}\left(\mu_{Q_{i}}\right)$, is the family of measures $v(A)=\int_{A} f(x) \mu_{Q_{i}}(d x), \forall A \in S$, that $\int|f(x)|^{2} \mu_{Q_{i}}(d x)<+\infty$ and $\|v\|_{H_{2}\left(\mu_{Q_{i}}\right)}=\left(\int|f(x)|^{2} \mu_{Q_{i}}(d x)\right)^{\frac{1}{2}}$.

Theorem 2. Let $M_{H}=\underset{i \in I}{\oplus} H_{2}\left(\mu_{Q_{i}}\right)$,

Be a Hilbert space of measures. $\mathrm{E}$ be the complete separable metric space. $\mathrm{S}$ be the borel $\sigma$-algebra in $\mathrm{E}$ and cardI $\leq 2^{\chi_{0}}$. Then in the theory (ZFC)\&(MA) the Gaussian stationary orthogonal statistical structures $\left\{E, S, \mu_{Q_{i}}, i \in I\right\}$, admits a consistent estimators of parameters $i \in I$ if and only if the correspondence $f \leftrightarrow \psi_{f}$, given by the equality $\int f(x) v(d x)=\left(\psi_{f}, v\right), \forall v \in M_{H}$ be one-to-one. Denote by $F=F\left(M_{H}\right)$ the set of real functions $\mathrm{f}$ for which $=\int_{E} f(x) \mu_{Q_{i}}(d x)$ is defined $\forall \mu_{Q_{i}} \in M_{H}$.

Proof.Necessity. The existence of a consistent estimator $\delta:(E, S) \rightarrow(I, B(I))$ of the parameter $Q_{i} \in I$ implies that $(\forall i)\left(i \in I \rightarrow \bar{\mu}_{i}\left(\left\{x: \delta(x)=Q_{i}\right\}\right)=1\right)$.

Setting $X_{i}=\left\{x: \delta(x)=Q_{i}\right\}$ for $i \in I$, we get:

1) $\bar{\mu}_{Q_{i}}\left(X_{i}\right)=\bar{\mu}_{i}\left(\left\{x: \delta(x)=Q_{i}\right\}\right)=1$ for $i \in I$;

2) $X_{Q_{i_{1}}} \cap X_{Q_{i_{2}}}=\varnothing$ for all different parameters $Q_{i_{1}}$ and $Q_{i_{2}}$ from I because $\left(X_{Q_{i_{1}}}=\left\{x: \delta(x)=Q_{i_{1}}\right\}\right) \cap\left(\left\{x: \delta(x)=Q_{i_{2}}\right\}=X_{Q_{i_{2}}}\right)=\varnothing$;

3) $\bigcup_{i \in I} X_{Q_{i_{1}}}=(\{x: \delta(x)=I\})=E$.

Therefore a statistical structure $\left\{E, S, \mu_{Q_{i}}, i \in I\right\}$ is strongly separable, so there exists S-measurable sets $\left\{X_{Q_{i}}\right\}_{i \in I}$ such that $\mu_{Q_{i}}\left(X_{Q_{j}}\right)=\left\{\begin{array}{l}1, \text { if } i=j \\ 0, \text { if } i \neq j\end{array}\right.$

Let function $I_{X_{Q_{i}}}(x) \in F$ be corresponded with $\mu_{Q_{i}} \in H_{2}\left(\mu_{Q_{i}}\right)$.

Then $\int I_{X_{Q_{i}}}(x) \mu_{Q_{i}}(d x)=\int I_{X_{Q_{i}}}(x) I_{X_{Q_{i}}} \mu_{Q_{i}}(d x)=\left(\mu_{Q_{i}}, \mu_{Q_{i}}\right)$. 
Let function $f_{\psi_{1}}(x)=f_{1}(x) \cdot I_{X_{\theta_{i}}}(x)$ be corresponded with $\psi_{1} \in H_{2}\left(\mu_{Q_{i}}\right)$. Then any $\psi_{2} \in H_{2}\left(\mu_{Q_{i}}\right) \cdot \int f_{\psi_{1}}(x) \psi_{2}(x) \mu_{Q_{i}}(d x)=\int f_{1}(x) f_{2}(x) I_{Q_{i}}(x) \mu_{Q_{i}}(d x)=\int f_{1}(x) f_{2}(x) \mu_{Q_{i}}(d x)=\left(\psi_{1}, \psi_{2}\right)$. Let function $f(x)=\sum_{i \in I_{f}} g_{i}(x) I_{X_{Q_{i}}}(x) \in F$ be corresponded with the measure $v \in M_{H}$ with the following from $\quad v=\sum_{i \in I_{f}} \int g_{i}(x) \mu_{Q_{i}}(d x), \quad$ then $\forall v_{1} \in M_{H}, v_{1}=\sum_{i \in I_{1}} \int g_{i}^{1}(x) \mu_{Q_{i}}(d x), \quad$ we $\quad$ have $\int f(x) v_{1}(d x)=\int \sum_{i \in I_{f} \cap I_{1}} g_{i}(x) g_{i}^{1}(x) \mu_{Q_{i}}(d x)=\sum_{i \in I_{f} \cap I_{1}} g_{i}(x) g_{i}^{1}(x) \mu_{Q_{i}}(d x)=\left(v_{1}, v\right)$.

From the proven theorem it follows that the above-indicated correspondence puts some function $f \subset F\left(M_{H}\right)$ into correspondence to each $\psi_{f} \in M_{H}$ If in $F\left(M_{H}\right)$ we identify the functions coinciding with respect to the measure $\left\{\mu_{Q_{i}}, i \in I\right\}$ then the correspondence will be bijective.

So the necessity is proved.

Sufficiency. Let $f \subset F\left(M_{H}\right)$ is corresponded with $\mu_{Q_{i}} \in M_{H}$ for which $\int f(x) \mu_{Q}(d x)=\left(\mu_{Q_{i}}, \mu_{Q}\right)$, then $\quad \mu_{Q_{1}}, \mu_{Q_{2}} \in M_{H} \quad$ we have $\int f_{Q_{1}}(x) \mu_{Q_{2}}(d x)=\left(\mu_{Q_{1}}, \mu_{Q_{2}}\right)=\int f_{1}(x) f_{2}(x) \mu_{Q_{1}}(d x)=\int f_{Q_{1}}(x) f_{2}(x) \mu_{Q_{1}}(d x)$,

So $f_{Q_{1}}=f_{1}$ for almost with respect to measure $\mu_{Q_{1}}$ every and $f_{Q_{1}}(x)>0, \int f_{Q_{1}}^{2}(x) \mu_{Q_{1}}(d x)<+\infty$, $\mu_{Q_{i}}^{*}=\int f_{Q_{i}}(x) \mu_{Q_{i}}(d x) \quad$ then $\quad \int f_{Q_{i}}^{*}(x) \mu_{Q_{j}}(d x)=\left(\mu_{Q_{i}}, \mu_{Q_{j}}\right)=0 \quad \forall i \neq j . \quad$ On $\quad$ other $\mu_{Q_{i}}\left(E-X_{Q_{i}}\right)=0, \quad X_{Q_{i}}=\left\{x: f_{Q_{i}}^{*}(x)>0\right\}$.

Hence it follows that $\mu_{Q_{i}}\left(X_{Q_{j}}\right)=\left\{\begin{array}{l}1, \text { if } i=j \\ 0, \text { if } i \neq j\end{array}\right.$, the statistical structure $\left\{E, S, \mu_{Q_{i}}, i \in I\right\}$ is weakly separable. Represent $\left\{\mu_{Q_{i}}, i \in I\right\}, \operatorname{cardI} \leq 2^{\chi_{0}}$ as an inductive sequence $\mu_{Q_{i}}<\omega_{1}$, where $\omega_{1}$ denotes the first ordinal number of the power of the set I.

Sense the statistical structure $\left\{E, S, \mu_{Q_{i}}, i \in I\right\}$ is weakly separable, there exists a family S-measurable sets $\left\{X_{Q_{i}}, i \in I\right\}$ such that the following are fulfilled: $\mu_{Q_{i}}\left(X_{Q_{j}}\right)=\left\{\begin{array}{l}1, \text { if } i=j \\ 0, \text { if } i \neq j\end{array}\right.$ for all $i \in\left[0 ; \omega_{1}\right)$

We define $\omega_{1}$ sequence of parts $Z_{Q_{i}}$ of the space $E$ so that the following relations are fulfilled:

1) $Z_{Q_{i}}$ is Bozel subset in $\mathrm{E}$ for all $i<\omega_{1}$;

2) $Z_{Q_{i}} \subset X_{Q_{i}}$ for all $i<\omega_{1}$;

3) $Z_{Q_{i}} \cap Z_{Q_{j}}=\varnothing$ for all $i<\omega_{1}, j<\omega_{1}, i \neq j$

4) $\mu_{Q_{i}}\left(Z_{Q_{i}}\right)=1$ for all $i<\omega_{1}$.

Assume that $Z_{Q_{0}}=X_{Q_{0}}$. Let further the partial sequence $\left\{Z_{Q_{j}}\right\}_{j<i}$ be already defined for $i<\omega_{1}$. It is clear, that $\mu^{*}\left(\bigcup_{j<i} Z_{Q_{j}}\right)=0$ (see [4]). Thus there exists a Borel subset $Y_{Q_{i}}$ of the space E such that the following relations are valid: $\bigcup_{j<i} Z_{Q_{J}} \subset Y_{Q_{i}}$ and $\mu\left(Y_{Q_{i}}\right)=0$ Assume $Z_{Q_{i}}=X_{Q_{i}}-Y_{Q_{i}}$, thereby the $\omega_{1}$ sequence of $\left\{Z_{Q_{i}}\right\}_{i<\omega_{1}}$ disjunctive measurable subsets of space $\mathrm{E}$ is constructed. Therefore $\mu_{Q_{i}}\left(Z_{Q_{i}}\right)=1$ for all $i<\omega_{1}$. 
A statistical structure $\left\{E, S, \mu_{Q_{i}}, i \in I\right\}, \quad c a r d I \leq 2^{\chi_{0}}$ is strongly separated there exists a family $\left\{Z_{Q_{i}}\right\}_{i \in I}$ of elements of $\sigma$-algebra $S_{1}=\bigcap_{i \in I} \operatorname{dom}\left(\bar{\mu}_{Q_{i}}\right)$ such that:

1. $\bar{\mu}_{Q_{i}}\left(Z_{Q_{i}}\right)=1, \quad \forall i \in I$

2. $Z_{Q_{i}} \cap Z_{Q_{j}}=\varnothing$ for all different parameters i and $\mathrm{j}$ for I;

3. $\bigcup_{i \in I} Z_{Q_{i}}=E$.

For $x \in E$, we put $\delta(x)=Q_{i}$, where $Q_{i}$ is unique parameter from the set I for which $x \in Z_{Q_{i}}$. The existence of such a unique parameter I can be proved by using conditions (2),(3).

Now let $Y \in B(I)$. Then $\{x: \delta(x) \in Y\}=\bigcup_{i \in Y} Z_{Q_{1}}$. We have to show that $\{x: \delta(x) \in Y\}=\operatorname{dom}\left(\bar{\mu}_{Q_{i_{0}}}\right)$ for each $Q_{i_{0}} \in I$.

If $Q_{i_{0}} \in Y$ then $\{x: \delta(x) \in Y\}=\bigcup_{i \in Y} Z_{Q_{i}}=Z_{Q_{i 0}} \cup\left(\bigcup_{i \in Y-\left\{Q_{i_{0}}\right\}_{i}} Z_{Q_{i}}\right) \subseteq \operatorname{dom}\left(\bar{\mu}_{Q_{i_{0}}}\right)$.

On the other hand, the validity of the condition $\left(\bigcup_{i \in Y-\left\{Q_{Q_{0}}\right\}_{Q_{i}}} Z_{\subseteq}\left(E-Z_{Q_{i 0}}\right)\right.$ implies that $\bar{\mu}_{Q_{i_{0}}}\left(\bigcup_{i \in Y-\left\{Q_{i_{0}}\right\}} Z_{Q_{i}}\right)=0$. The latter equality yields that $\left(\bigcup_{i \in Y-\left\{Q_{i_{0}}\right\}} Z_{Q_{i}}\right) \in \operatorname{dom}\left(\bar{\mu}_{Q_{i_{0}}}\right)$.

Since $\operatorname{dom}\left(\bar{\mu}_{Q_{i_{0}}}\right)$ is $\sigma$-algebra, we deduce that $\{x: \delta(x) \in Y\}=Z_{Q_{i 0}} \cup\left(\bigcup_{i \in Y-\left\{Q_{i 0}\right.} Z_{Q_{i}}\right) \in \operatorname{dom}\left(\bar{\mu}_{Q_{i_{0}}}\right)$.

If $Q_{i 0} \notin Y$, then $\{x: \delta(x) \in Y\}=\bigcup_{i \in Y} Z_{Q_{i}} \subseteq E-Z_{Q_{Q_{0}}}$ and we claim that $\bar{\mu}_{Q_{i 0}}(\{x: \delta(x) \in Y\})=0$. The letter relation implies that $\{x: \delta(x) \in Y\} \in \operatorname{dom}\left(\bar{\mu}_{Q_{i_{0}}}\right)$.

Thus we have shown the validity of the condition $\{x: \delta(x) \in Y\} \in \operatorname{dom}\left(\bar{\mu}_{Q_{i 0}}\right)$ for any arbitrary $Q_{i 0} \in I$ Hence $\{x: \delta(x) \in Y\} \in \bigcap_{i \in I} \operatorname{dom}\left(\bar{\mu}_{Q_{i}}\right)=S_{1}$ we have shown that the $\operatorname{map} \delta:(E, S) \rightarrow(I, B(I))$ is measurable map.

Since $\mathrm{B}(\mathrm{I})$ contains all singletons of $\mathrm{I}$, we claim that $(\forall i)\left(i \in I \rightarrow \bar{\mu}_{Q_{i}}\left\{x: \delta(x)=Q_{i}\right\}\right)=\bar{\mu}_{Q_{i}}\left(Z_{Q_{i}}\right)=1$

Theorem 2 is proved.

\section{References}

[1] I. Ibramhalilov, A. Skorokhod. Consistent estimates of parameters of random processes. Naukova Dumka. Kiev (1980).

[2] T. Jech. Set Theory. Springer, Berlin (2003).

[3] A. Kharazishvili. Topologic aspects of measure theory. Naukova Dumka. Kiev (1984).

[4] Z. Zerakidze. On weakly divisible and divisible families of probability measures. Bull Acad.Sci.Georgian SSR 113(2) 273-275 (1984).

[5] Z. Zerakidze. About the conditions equivalence of Gaussian measures corresponding to homogeneous fields. Works of Tbilisi State University. Vol 2 215-220 (1969).

[6] Z. Zerakidze. On consistent estimators for families of probability measures.5-th Japan - USSR Symposium on Probability Theory. Kyoto, pp 62-63 (1986).

[7] Z. Zerakidze Hilbert space of measures. Ukr. Math. Journal 38 (2) pp 147-153 Kiev (1986).

[8] Z. Zerakidze, M. Mumladze. Statistical structures and consistent criteria for checking hypotheses. Lambert Academic Publishing. ISBN: 978-2-659-69689-3 Saarbzucken Deutschland (2015).

[9] G. Pantsulaia. On orthogonal families of probability measures (in Russian) Trans.GP1-8 (3501-pp 106-112) (1989).

[10] G. Pantsulaia. On separation properties for families of probability measures. Georg. Math Journal 10(2) pp. 335-342 (2003).

[11] I. Rozanov, I. Ibragimov. Gaussian random processes. Nauka. Moscow (1970). 